Research Paper

\title{
Zika Virus Envelope Protein induces G2/M Cell Cycle Arrest and Apoptosis via an Intrinsic Cell Death Signaling Pathway in Neuroendocrine PC12 Cells
}

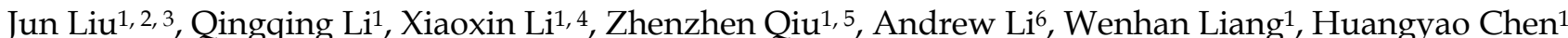
Xiangsheng Cai ${ }^{1,7}$, Xinglu Chen ${ }^{1,7}$, Xinyue Duan ${ }^{1}$, Jingjing $\mathrm{Li}^{1}$, Wangsheng Wu ${ }^{1,8}$, Mingyu Xu ${ }^{1}$, Yingying $\mathrm{Mao}^{1}$, Huiying Chen ${ }^{1}$, Jinlong $\mathrm{Li}^{1}$, Weiwang $\mathrm{Gu}^{2,9,10,11^{\bowtie}}$, Hongwei $\mathrm{Li}^{1 凶}$

1. School of Laboratory Medicine and Biotechnology, Southern Medical University, Guangzhou, China.

2. Institute of Comparative Medicine and Center of Laboratory Animals, Southern Medical University, Guangzhou, China.

3. Dermatology Hospital of Southern Medical University, Guangzhou, China.

4. Division of Life Science, State Key Laboratory of Molecular Neuroscience, The Hong Kong University of Science and Technology, Hong Kong, China.

5. Guangzhou Bioneeds Biotechnology Co., Ltd, Guangzhou, China.

6. Department of Biomedical Engineering, The Johns Hopkins University School of Medicine, Baltimore, USA

7. Clinical Laboratory, The First Affiliated Hospital of Guangdong Pharmaceutical University, Guangzhou, China.

8. Animal Science and Technology College, Jilin Agricultural University, Changchun, China.

9. Pearl Lab Animal Science and Technology Co. Ltd, Dongguang, China.

10. School of Chemical and Environmental Engineering, Wuyi University, Jiangmen, China.

11. International Healthcare Innovation Institute, Jiangmen, China.

$\square$ Corresponding authors: Weiwang Gu, MS, Institute of Comparative Medicine and Center of Laboratory Animals, Southern Medical University, 1023 South Shatai Road, Guangzhou, Guangdong 510515, China. Phone: 86-20-61648043; Fax: 86-20-61648043; E-mail: guww100@163.com; Hongwei Li, Ph.D., School of Laboratory Medicine and Biotechnology, Southern Medical University, 1023 South Shatai Road, Guangzhou, Guangdong 510515, China. Phone: 86-20-61648555; Fax: 86-20-61648555; E-mail: lihw@smu.edu.cn

(c) Ivyspring International Publisher. This is an open access article distributed under the terms of the Creative Commons Attribution (CC BY-NC) license (https://creativecommons.org/licenses/by-nc/4.0/). See http://ivyspring.com/terms for full terms and conditions.

Received: 2018.03.31; Accepted: 2018.05.13; Published: 2018.06.08

\begin{abstract}
Clinical evidence suggests that there exists a strong correlation between Zika virus (ZIKV) infection and abnormal development of the nervous system. However, the underlying mechanisms remain to be elusive. In this study, recombinant lentiviral vectors coding for ZIKV structural proteins and truncations (prM-Env, M-Env and Env) were transduced into PCl2 cells. Envelope (Env) overexpression induced significant inhibition of proliferation and triggered G2/M cell cycle arrest and apoptosis in $\mathrm{PC} 12$ cells. Flow cytometry and western blot analysis showed that the apoptosis was associated with up-regulation of both $\mathrm{p} 53$ and $\mathrm{p} 21^{\mathrm{Cip} / \text { Wafl }}$ and down-regulation of cyclin B1. Presence of aberrant nuclei clusters were confirmed by immunofluorescence staining analysis. The data indicate that overexpression of prM-Env, M-Env or Env led to apoptosis via an intrinsic cell death signaling pathway that is dependent on the activation of caspase- 9 and caspase- 3 and accompanied by an increased ratio of Bax to $\mathrm{Bcl}-2$ in transduced $\mathrm{PCl} 2$ cells. In summary, our results suggest that ZIKV Env protein causes apoptosis in $\mathrm{PCl} 2$ cells via an intrinsic cell death signaling pathway, which may contribute to ZIKV-induced abnormal development of the nervous system.
\end{abstract}

Key words: ZIKV, envelope protein, G2/M arrest, mitotic catastrophe, apoptosis

\section{Introduction}

The Zika virus (ZIKV), a member of the Flaviviridae family, is mainly transmitted by Aedes aegypti mosquitos [1]. ZIKV infections have become a major public health problem in the tropical and sub-tropical regions of the world with significant burden to ZIKV-infected patients and society as a whole [2-4]. In Latin American countries, public recommendations to couples are to postpone pregnancy from six months to two years in ZIKV epidemic areas [5-7].

In ZIKV epidemic areas, most noticeably Brazil, a sharp increase in the incidence of microcephaly was 
observed amongst newborns [8-10]. Additionally, a high incidence of Guillain-Barré syndrome was reported in Colombia [11] where ZIKV infections were highly prevalent from November of 2015 to March of 2016. Epidemiological data also showed that in 401 cases of ZIKV-associated secondary nervous system disease, $67 \%$ patients were diagnosed with Guillain-Barré syndrome [12]. These reports indicated that there exists a correlation between ZIKV infection and Guillain- Barré syndrome [13]. Recently, Souza, B.S and colleagues also found that ZIKV infection induces mitosis abnormalities and apoptotic cell death in human neural progenitor cells reinforcing the link between ZIKV and developmental neurological disorders abnormalities in the developing human brain, including such as microcephaly [14]. Despite much attention, however, the underlying mechanisms explaining this link remain elusive [15].

The ZIKV genome is presented as positive sense ssRNA encoding three structural proteins (Core (C), premembrane/membrane ( $\mathrm{prM} / \mathrm{M})$ and envelope (Env)) and seven non-structural proteins (NS1, NS2A, NS2B, NS3, NS4A, NS4B, NS5) [16]. The prM/M protein acts as a chaperone for the correct folding of the Env protein during virus assembly and maturation [17, 18]. The Env protein plays a crucial role in viral infection and replication of Flavivirus [19]. Studies also have shown that the Env protein can induce cell-cycle G2/M accumulation [20]. In the present study, we first explored the effects of ZIKV prM-Env and two truncations (M-Env and Env) in neuroendocrine PC12 cells. Our results confirmed the effect of ZIKV Env protein in interfering with the cell cycle suggesting a role in abnormal nervous system development. The cell cycle arrest and apoptosis caused by Env was associated with increases in the expression levels of p21 Cip1/Waf1 and p53, as well as decreases in the levels of cyclin B1. Meanwhile, the apoptosis induced by Env was confirmed by upregulation of cleaved caspase- 9 , and caspase- 3 in PC12 cells.

\section{Materials and Methods}

\section{Cells and Plasmids}

Human embryonic kidney (HEK) 293T cells and rat pheochromocytoma PC12 cells (ATCC, Manassas, VA) were cultured in Dulbecco's modified Eagle's medium (DMEM) (Hyclone, Logan, UT) supplemented with 10\% fetal bovine serum (FBS) (Hyclone, US), 100 units $/ \mathrm{ml}$ penicillin, $100 \mathrm{mg} / \mathrm{ml}$ streptomycin (Invitrogen, US) and maintain in $5 \% \mathrm{CO}_{2}$ at $37^{\circ} \mathrm{C}$.

pLV-eGFP containing Cytomegalovirus (CMV) promoter was constructed in this lab. The packaging plasmids pMD2.G and psPAX2 were obtained from
Dr. Junming Yue (Department of Pathology, University of Tennessee Health Science Center). The cDNA fragment of the ZIKV prM-Env (Haiti strain, GenBank: KU509998.3) was obtained by chemical synthesis (IGE Biotechnology, China) and the cDNA fragments of M-Env and Env were obtained using PCR method. Primers are listed in Table 1. A six-histidine coding region was fused to the $3^{\prime}$ end of each cDNA fragment. All cDNA fragments were cloned into the lentiviral expression plasmid pLV-eGFP. The resulting plasmids were designated as pLV-eGFP-ZIKV-prM-Env, pLV-eGFP-ZIKV-M-Env and pLV-eGFP-ZIKV-Env, which were identified by restriction enzyme digestion and partial sequencing.

Table 1 Gene-specific primer sets used in this study

\begin{tabular}{|c|c|c|c|}
\hline $\begin{array}{l}\text { Gene } \\
\text { name }\end{array}$ & Prime name & Sequence $\left(5^{\prime}-3^{\prime}\right)$ & $\begin{array}{l}\text { Product } \\
\text { size (bp) }\end{array}$ \\
\hline \multirow[t]{2}{*}{ M-Env } & M-Env-F & GCTAGCGCTGTGACGCTCCCC & 1758 \\
\hline & M-Env-R & ACGCGTTTAATGGTGATGGTGATG & \\
\hline \multirow[t]{2}{*}{ Env } & Env-F & GCTAGCATCAGGTGCATAGGAG & 1533 \\
\hline & Env-R & ACGCGTTTAATGGTGATGGTGATG & \\
\hline \multirow[t]{2}{*}{ Bax } & BAX-F & GTCAGCTGCCACACGGAAG & 81 \\
\hline & BAX-R & AACAACATGGAGCTGCAGAGGAT & \\
\hline \multirow[t]{2}{*}{$B c l-2$} & Bcl-2-F & TCAGAGACAGCCAGGAGAAATCA & 131 \\
\hline & Bcl-2-R & CCTGTGGATGACTGAGTACCTGAA & \\
\hline \multirow[t]{2}{*}{ Cyclin B1 } & Cyclin B1-F & GCGTGTGCCTGTGACAGTTA & 135 \\
\hline & Cyclin B1-R & CCTAGCGTTTTTGCTTCCСTT & \\
\hline \multirow[t]{2}{*}{ p53 } & p53-F & AGCCAGACAGATCTTGTGAG & 134 \\
\hline & p53-R & TCCCGCACGGCAAACTTTATC & \\
\hline \multirow[t]{2}{*}{$p 21$} & $\mathrm{p} 21-\mathrm{F}$ & CCTGGTGATGTCCGACCTG & 103 \\
\hline & $\mathrm{p} 21-\mathrm{R}$ & CCATGAGCGCATCGCAATC & \\
\hline \multirow[t]{2}{*}{$\beta$-actin } & $\beta$-actin-F & AAGATTCGAGAAGAATACCCTGA & 121 \\
\hline & $\beta$-actin-R & CTACCAACTGGTGGACGGACA & \\
\hline
\end{tabular}

\section{Generation of recombinant lentiviral vectors}

The recombinant lentiviruses LV-ZIKV-prMEnv, LV-ZIKV-M-Env, LV-ZIKV-Env and LV-eGFP were packaged and titered as described previously $[21,22]$.

\section{Lentiviral transduction in vitro}

A total of $10^{5}$ PC12 cells / well were prepared in a 6-wells plate. On the following day, the cells in each well were transduced with packaged recombinant lentiviruses at a multiplicity of infection (MOI) of 100 in DMEM medium containing 10\% FBS with $6 \mu \mathrm{g} / \mathrm{ml}$ hexadimethrine bromide (Polybrene, Sigma, US). After $24 \mathrm{~h}$, transduction media was replaced with fresh DMEM with 10\% FBS and incubated for $24 \mathrm{~h}$ at $37^{\circ} \mathrm{C}$ and $5 \% \mathrm{CO}_{2}$.

\section{Cell proliferation assay}

For detection of cell proliferation, MTT assay were performed at $48 \mathrm{~h}$ post-transduction. Total $10 \mu \mathrm{l}$ of MTT regent (Sigma-Aldrich, US) at the concentration of $5 \mathrm{mg} / \mathrm{ml}$ was added to each well. Following $3 \mathrm{~h}$ of incubation, $100 \mu \mathrm{l}$ of dimethyl sulphoxide 
(DMSO) detergent (Sigma-Aldrich, US) was added to each well. The plate was then incubated on a shaker at $4{ }^{\circ} \mathrm{C}$ for $2 \mathrm{~h}$, and absorbance was measured at a 570 $\mathrm{nm}$ with a microplate reader (Bio-Rad, US).

\section{Cell cycle analyses}

For detection of cell cycle, lentiviral transducedcells were washed with phosphate buffer saline (PBS) and fixed with $70 \%$ ice cold ethanol at $4^{\circ} \mathrm{C}$ for overnight, followed by treatment with PI (SigmaAldrich, US) solution at a final concentration of 50 $\mu \mathrm{g} / \mathrm{ml}$. The DNA content was measured using flow cytometry (Beckman Coulter, Epics XL).

\section{Immunofluorescence analyses}

PC12 cells were collected $48 \mathrm{~h}$ post-transfection and fixed with $4 \%$ paraformaldehyde (Solarbio, China) for $20 \mathrm{~min}$, permeabilized with $0.1 \%$ Triton X-100 (Biosharp, China) for $15 \mathrm{~min}$. Immunostaining was then done on the fixed cells as detailed previously [23] using a monoclonal antibody against $6 \times$ His tag (1: 500, Abcam, US) followed by Alexa Fluor 633 anti-mouse IgG (1:1,000; ThermoFisher Scientific, US) as the secondary antibody. Nuclei DNA staining was performed with 4', 6-diamidino-2phenylindole (DAPI, Sigma-Aldrich, US). Images were captured using a fluorescence microscope (Nikon, Japan).

\section{Annexin V-Alexa 647 apoptosis assay}

For detection of cell apoptosis, lentiviraltransduced PC12 cells were collected and incubated with $1 \mu \mathrm{g} / \mathrm{ml}$ Annexin V-Alexa 647 (Abcam, US) and $2.5 \mu \mathrm{g} / \mathrm{ml}$ propidium iodide (PI, Sigma-Aldrich, US) for $30 \mathrm{~min}$ at room temperature, and then analyzed using a BD FACSCanto II cytometer (Becton Dickinson). Specific inhibitors of caspase-3 (Ac-DEVD-CMK, Santa Cruz Biotechnology, US), caspase-8 (Z-LEHD-FMK, Abcam, US) and caspase-9 (Z-LEHD-FMK, Abcam, US) were used to confirm the assay specificity.

\section{Western blot analysis}

Western immunoblots were run as described previously [21]. Primary antibodies and their sources were as follows. Anti-total p53, anti-cyclin B1, anti-Bax, anti-Bcl-2, anti-CDK1, anti-phospho-CDK1, anti-activated caspase 8 , anti-activated caspase 9 , and anti-activated caspase 3 were from Cell Signaling Technology. Anti-ZIKV Env monoclonal antibody was purchased from Zoongen Co., Ltd (Beijing, China). Anti-p21Cip1/Waf1 and anti- $6 \times$ His tag was from Abcam. Anti- $\beta$-actin and the secondary antibodies horseradish peroxidase-conjugated anti-rabbit IgG and anti-rabbit IgG were from Sigma-Aldrich. Anti-goat IgG was from Santa Cruz Biotechnology.

\section{Quantitative real-time RT-PCR analysis}

Total RNA was isolated from transduced PC12 cells using TRIzol ${ }^{\circledR}$ (Sigma, US), and then converted into cDNA with iScript ${ }^{\mathrm{TM}}$ Select cDNA Synthesis Kit (Bio-Rad, US). Quantitative real-time RT-PCR was performed on an ABI 7500 real-time PCR system (Applied Biosystems, US) as described previously [23]. Primers for target genes (Bax, Bcl-2, Cyclin B1, $p 53, p 21$ and $\beta$-actin) are listed in Table 1 . The samples were quantified by the comparative $\triangle \triangle C_{\mathrm{T}}$ method by using rat $\beta$-actin as the internal standard.

\section{Statistical analysis}

All continuous variables are presented as means \pm SEM. Parametric data were analyzed using unpaired two-tailed $t$ tests, for comparisons between two groups, and 1-way ANOVA, followed by Bonferroni post hoc test for multiple-comparison test, using Prism 6.0 (GraphPad Software Inc, La Jolla, CA, USA). Values of $p<0.05$ were considered statistically significant.

\section{Results}

\section{Lentivirus- mediated overexpression of ZIKV prM-Env, M-Env and Env in PC12 cells}

Cell supernatants and cells were collected at 12, $24,48 \mathrm{~h}$ post-transduction to assess the expression of ZIKV prM-Env, M-Env and Env in transduced PC12 cells using Western blot analysis. The results showed that they were detectable as early as $24 \mathrm{~h}$ post-transduction in cell lysates (Figure 1A), but not in supernatants (data not shown). The expressions of prM-Env, M-Env and Env were further confirmed by indirect immunofluorescence at $48 \mathrm{~h}$ post-transduction. The results revealed that these proteins were localized in the nucleus and cytoplasm of transduced PC12 cells (Figure 1B).

\section{prM-Env, M-Env and Env inhibited PC1 2 cell growth and induced $G 2 / M$ arrest in lentivirus-transduced $P C 12$ cells}

MTT assays were performed to determine the effect of prM-Env, M-Env and Env on transduced PC12 cell growth. We demonstrated that lentivirusmediated overexpression of prM-Env, M-Env and Env significantly inhibited PC12 cells growth when compared with LV-eGFP transduced cells, but not amongst themselves (Figure 2A).

To examine the effect of prM-Env M-Env and Env on cell cycle, flow cytometric analysis was performed at $48 \mathrm{~h}$ post-transfection. The results showed that overexpression of prM-Env, M-Env and Env significantly increased the percentage of cells in the G2/M phase in transduced PC12 cells (Figure 2B). 
B
A

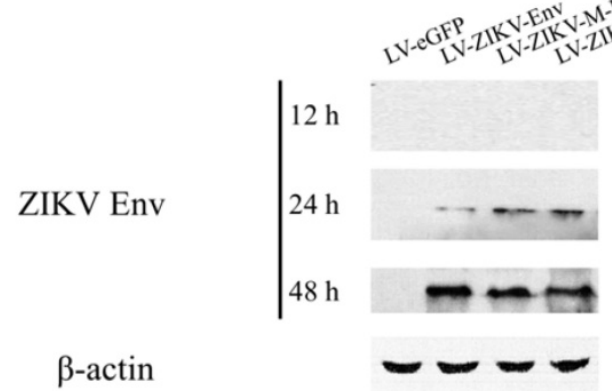

$\beta$-actin

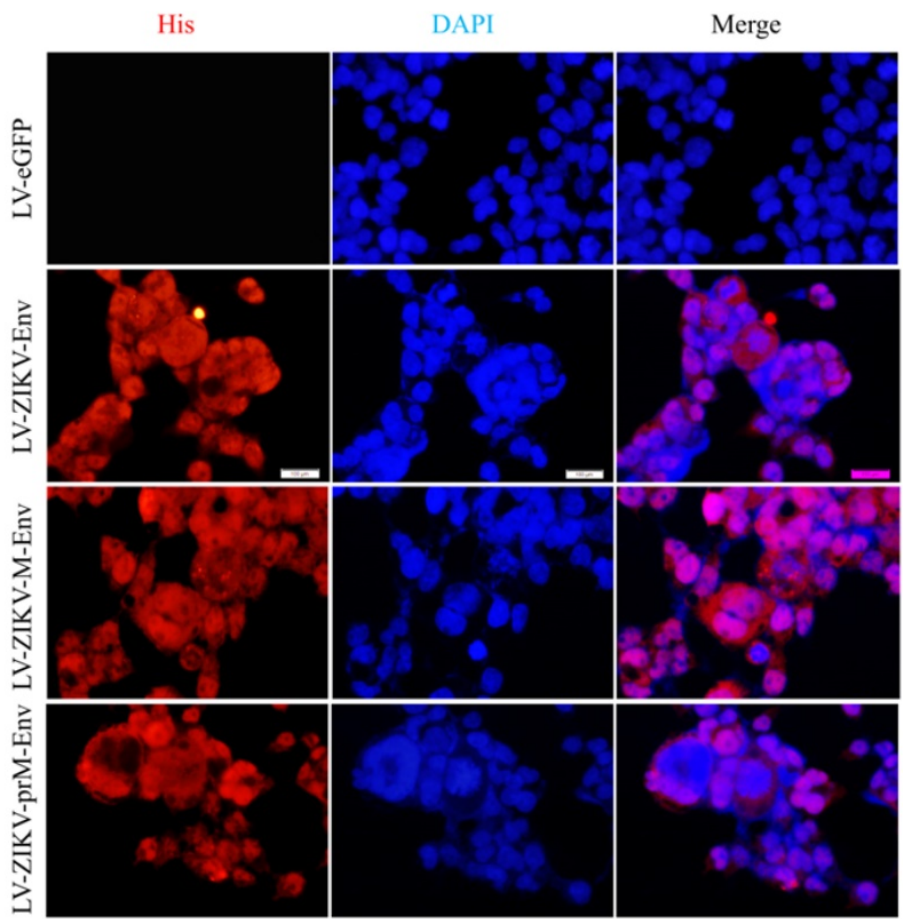

Figure 1. Lentiviral vectors-mediated ZIKV Env, M-Env and prM-Env overexpression in PC12 cells. Cells were infected with LV-ZIKV-prM-Env, LV-ZIKV-M-Env, LV-ZIKV-Env or LV-eGFP at an MOI of 100. (A) At 12, 24 and $48 \mathrm{~h}$ after transduction, cell protein extracts were subjected to Western blot analysis using an anti-Env antibody. (B) At $48 \mathrm{~h}$ after transduction, ZIKV Env, M-Env or prM-Env immunoreactivity was also detected using a mouse monoclonal anti-His antibody followed by anti-mouse Alexa Fluor 633-conjugated antibody (Scale bar= $100 \mu \mathrm{m})$.

The percentage of G2/M phase LV-ZIKV-prM-EnV, LV-ZIKV-M-Env and LV-ZIKV-Env transduced cells increased by $20 \%$ compared to that of LV-eGFP infected PC12 cells (Figure 2C). Additionally, no significant difference was observed in G2/M phase ratio among LV-ZIKV-prM-Env, LV-ZIKV-M-Env and LV-ZIKV-Env transduced PC12 cells. The Envinduced G2/M arrest in transduced PC12 cells was further confirmed by downregulation of G2/mitoticspecific cyclin B1 and inhibition phosphorylation of cell cycle kinase CDK1 (Figure 2D\&E).

\section{prM-Env, M-Env and Env induced mitotic catastrophe in lentivirus-transduced PC12 cells}

Aberrant nuclei clusters were observed in LVZIKV-prM-Env, LV-ZIKV-M-Env and LV-ZIKV-Envtransduced PC12 cells (Figure 3A). This indicated that prM-EnrEnv, M-Env and Env induced significant mitotic catastrophe in transduced PC12 cells. Approximately 12 to $15 \%$ of PC12 cells, expressing prM-Env M-Env or Env showed different abnormal nuclei compared to the control cells (Figure 3B).

\section{prM-Env, M-Env and Env induced apoptosis in lentivirus-transduced $P C 12$ cells via an intrinsic cell death signaling pathway}

We further examined whether prM-Env, M-Env and Env could elicit apoptosis in transduced PC12 cells since they caused the increment of G2/M phase and induction of mitotic catastrophe in these cells. Annexin $\mathrm{V}$, an endogenous $36-\mathrm{kDa}$ protein that binds to phosphatidylserine, have been identified and reported as potential apoptosis probes [24]. Annexin V-Alexa Fluor 647-conjugated staining was performed to evaluate apoptosis in PC12 cells at $48 \mathrm{~h}$ post-transduction. Flow cytometric results indicated a significantly higher proportion of annexin $\mathrm{V}$ positive 
A

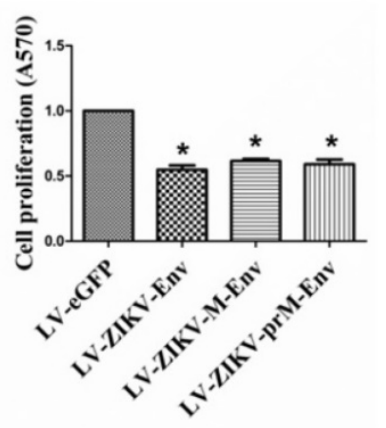

C

D
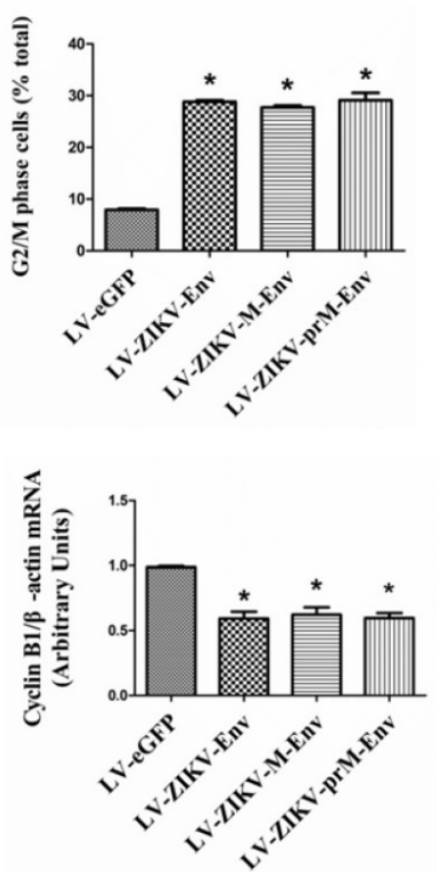

B
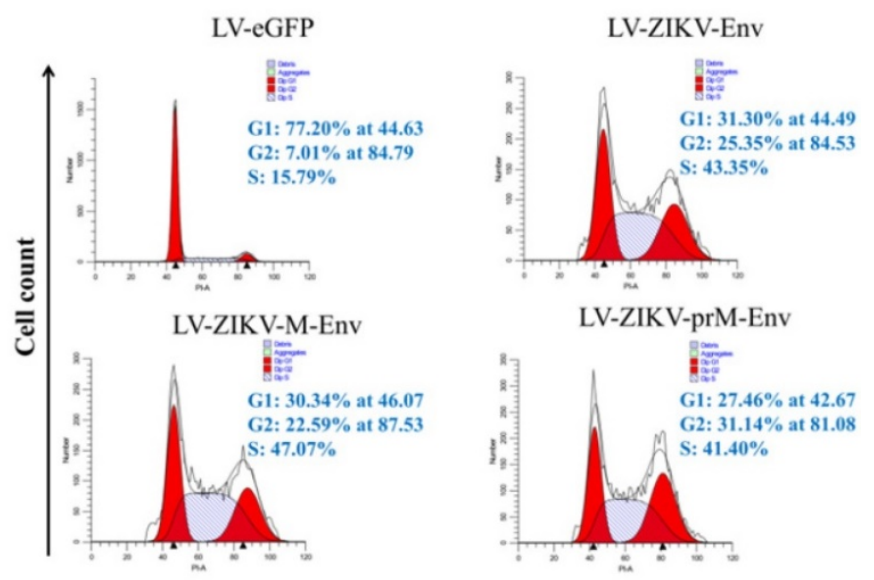

LV-ZIKV-prM-Env

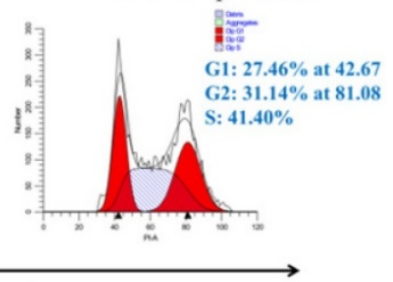

DNA content

E

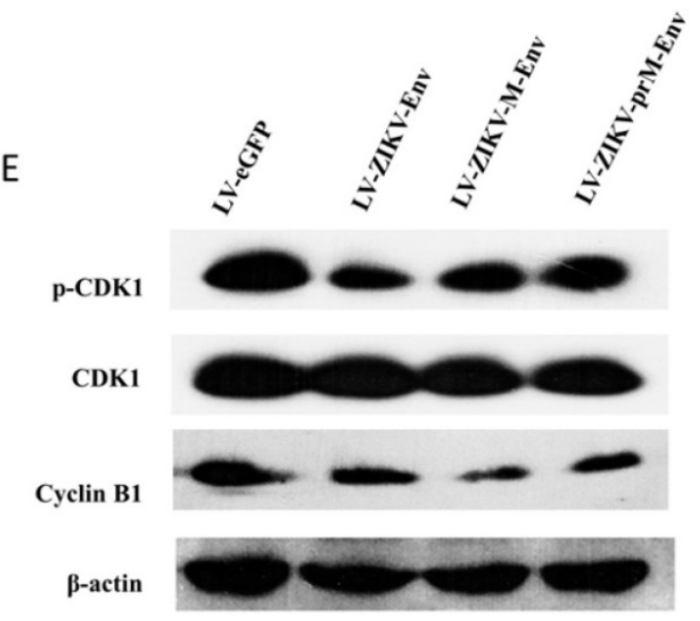

Figure 2. prM-Env, M-Env and Env inhibited $P C 12$ cell growth and induced $G 2 / M$ arrest in transduced $P C 12$ cells. $P C 12$ cells were transduced with LV-ZIKV-prM-Env, LV-ZIKV-M-Env, LV-ZIKV-Env or LV-eGFP at an MOI of 100. After $48 \mathrm{~h}$ of incubation, cells were collected and subjected to MTT assay, flow cytometric analysis, real-time RT-PCR or Western blot. (A) Cell proliferation determined by MTT assay. *: P $<0.05$ versus with LV-eGFP. (B) Representative data for lentivirus-induced G2/M cell cycle arrest measured by flow cytometry at $48 \mathrm{~h}$ after treatment. The $y$ axis represents the cell count, and the $x$ axis represents the DNA content. The experiments were performed at least three times. (C) Data showing percentages of PC12 cells in G2/M phase. *: P<0.05 versus with LV-eGFP. Data are representative of three independent experiments. (D) Cyclin B1 mRNA relative levels in transduced PC12 cells.*: P<0.05 versus with LV-eGFP. (E) Cyclin B1 and CDK1 expression in transduced PC12 cells. Data are representative of three experiments.

cells in the prM-Env, M-Env and Env expressing groups when compared to the control group (Figure $4 \mathrm{~A} \& 4 \mathrm{~B})$. Apoptosis associated proteins were then detected by western blot to explore the mechanism for Env-induced apoptosis. Results indicate that p53, p21 Cip1/Waf1, Bax, cleaved caspase-3, cleaved caspase8 , cleaved caspase- 9 were increased, while Bcl-2 was decreased in PC12 cells transduced with LV-ZIKV-prM-Env, LV-ZIKV-M-Env and LV-ZIKVEnv when compared with LV-eGFP (Figure 4C). The mRNA relative levels of $p 53, p 21_{\text {Cip1/Naf1 }, B a x}$, and $B c l-2$ were then measured by real-time RT-PCR in transduced PC12 cells. The results revealed significant up-regulation of $p 53, p 21^{\text {Cip } 1 / N a f 1}$, and Bax mRNA relative levels in LV-ZIKV-Env, LV-ZIKV-M-Env, and LV-ZIKV-prM-Env transduced PC12 cells while the mRNA levels of Bcl-2 (Figure 4D) in PC12 cells with
Env, M-Env and prM-Env overexpression were significantly lower than that of control PC12 cells.

Lastly, treatment of PC12 cells with the caspase- 3 inhibitor Ac-DEVD-CMK $(100 \mu \mathrm{mol} / \mathrm{L})$ significantly reduced the apoptosis elicited by LV-ZIKV-prM-Env, LV-ZIKV-M-Env or LV-ZIKV-Env transduction (Figure 5A \& 5B). Furthermore, similar inhibition of ZIKV Env-induced apoptosis was obtained by treatment of the PC12 cells with the caspase-9 inhibitor Z-LEHD-FMK (100 $\mu \mathrm{mol} / \mathrm{L})$ while no inhibition effects were observed with caspase-8 inhibitor Z-IETD-FMK $(100 \mu \mathrm{mol} / \mathrm{L})$. These data suggest the involvement of a caspase-9-mediated intrinsic signaling pathway followed by downstream activation of caspase- 3 in the apoptosis elicited by overexpression of ZIKV prM-Env, M-Env and Env in PC12 cells. 
A

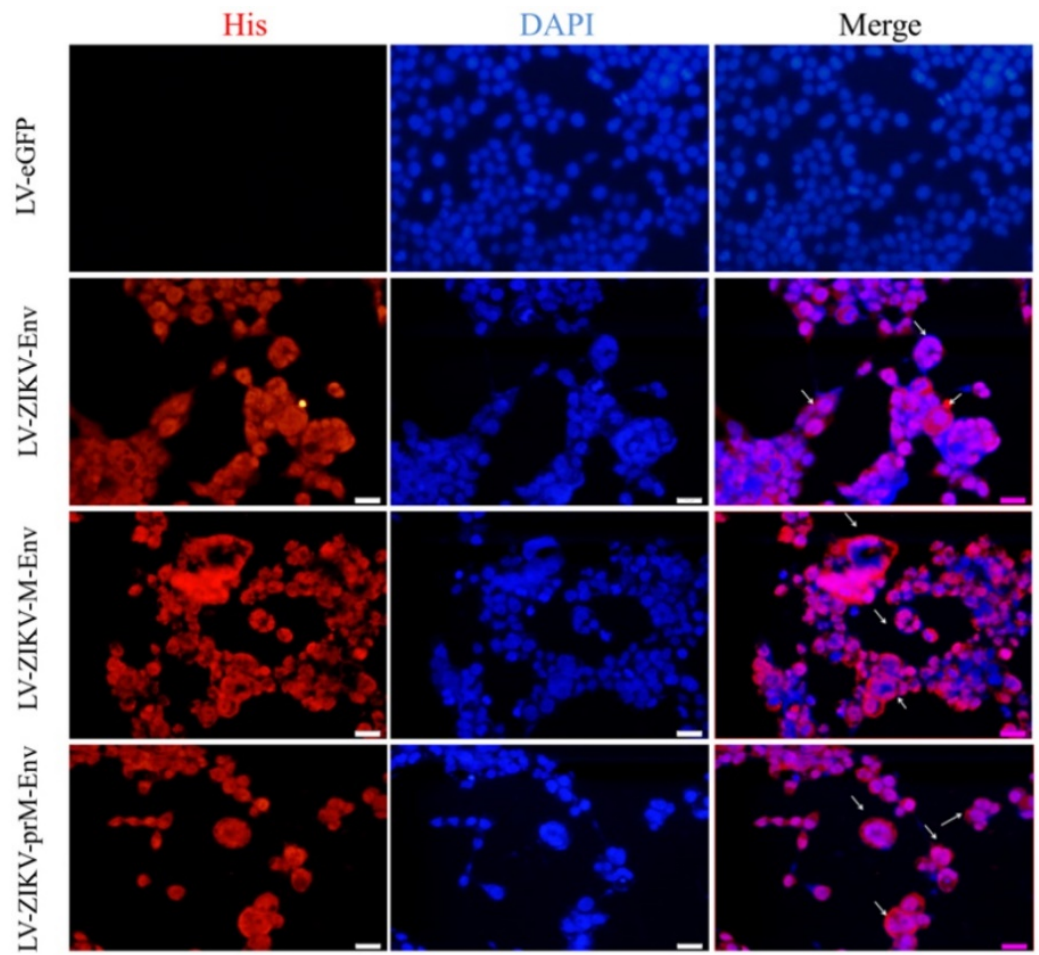

B

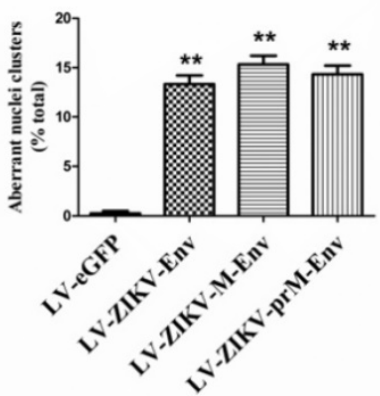

Figure 3. ZIKV Env, M-Env and prM-Env induced mitotic catastrophe in lentiviruses-transduced PC12 cells. (A) PC12 cells were transduced with LV-ZIKV-prM-Env, LV-ZIKV-M-Env, LV-ZIKV-Env or LV-eGFP at an MOI of 100, immunostained with an anti-His antibody followed by anti-mouse Alexa Fluor 633-conjugated antibody, and then counterstained with DAPI for nuclear staining (Scale bar $=100 \mu \mathrm{m}$ ). Mitotic catastrophe was characterized by the appearance of aberrant nuclei clusters. The number of aberrant nuclei clusters in each treatment condition was counted from 5 random fields per well by an individual who was blinded to the treatment. (B) Data are presented as a percent of the total number of cells on the dish. **: $P<0.01$ versus with LV-eGFP.

\section{Discussion}

The severe clinical symptoms caused by ZIKV infection have aroused world-wide attention. Significant breakthroughs and new questions have been made during the rapid attempt to understand the relationship between ZIKV and abnormal development of nervous system $[15,25,26]$. The neuronal apoptosis induced by ZIKV has been confirmed both in vitro [14] and in vivo [13, 27]. The data presented here indicate that overexpression of ZIKV prM-Env, M-Env and Env in PC12 cells induced inhibition of cell growth, G2/M arrest and mitotic catastrophe. Furthermore, the overexpression of these proteins led to apoptosis via an intrinsic cell death signaling pathway that is dependent on activation of caspase-9 and caspase-3. Finally, the apoptosis induced by ZIKV prM-Env, M-Env and Env overexpression was accompanied by up-regulation of both p53 and p21 Cip1/Waf1. Collectively, the observations presented here indicate the ability of increased ZIKV prM-Env, M-Env and Env expression to induce apoptosis in neuroendocrine PC12 cells in vitro suggesting that the ZIKV Env protein may play a crucial role in ZIKV-induced neuronal apoptosis.

The prM-Env protein of ZIKV is a major candidate antigen for vaccine development [28]. We had initially planned to produce this protein in HEK293T by transduction with LV-prM-Env, LV-MEnv and LV-Env but inadvertently observed significant apoptosis (data not shown). HEK293 cells are ubiquitously used for many purposes [29] but can also be used for analysis of neuronal synapse formation [30] and has even been argued that HEK293 cells are of neuronal lineage [31]. Our accidental finding in HEK293 cells led to this investigation of the 


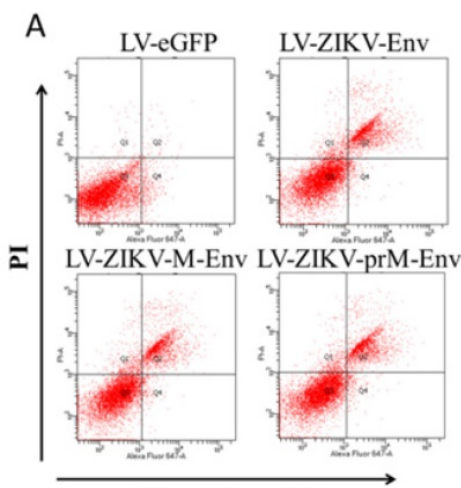

Alexa Fluor 647

B

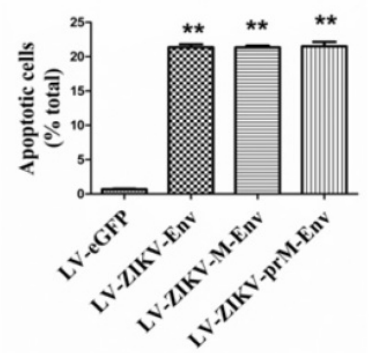

C

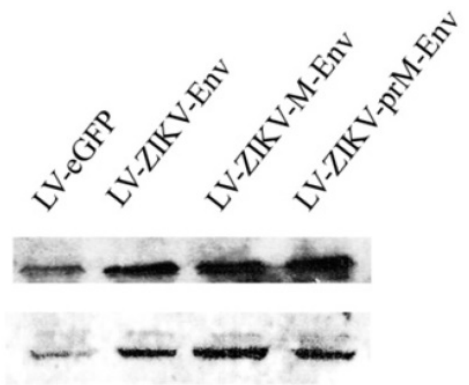

Cleaved Caspase-3

Cleaved Caspase-8

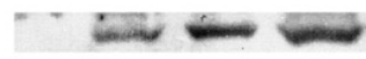

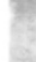

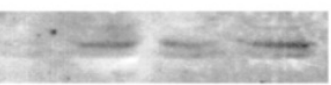

Cleaved Caspase-9

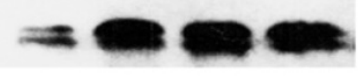

Bax

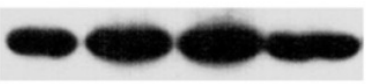

Bcl-2

$\beta$-actin

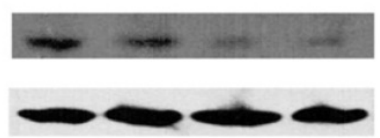

D
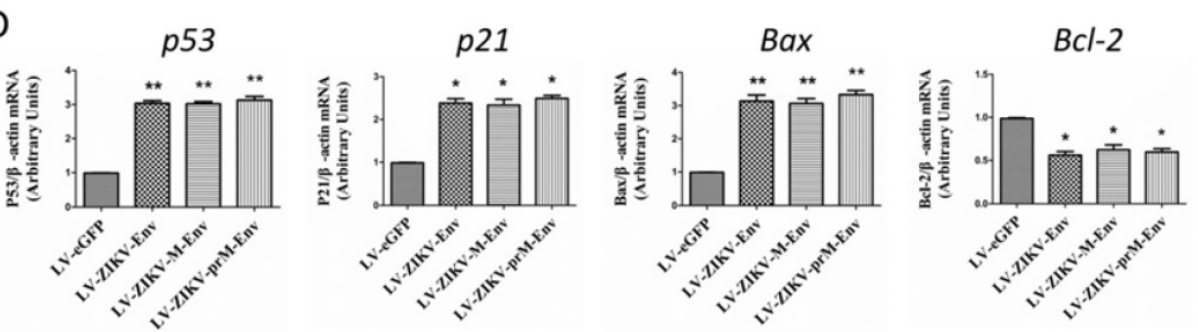

Figure 4. ZIKV Env, M-Env and prM-Env induced apoptosis in lentiviral vectors-transduced PC12 cells. PC12 cells were transduced with LV-ZIKV-prM-Env, LV-ZIKV-M-Env, LV-ZIKV-Env or LV-eGFP at a MOI of 100. (A) At $48 \mathrm{~h}$ post- transduction, indicated cells were collected and double-stained with Alexa Fluor 647 conjugated annexin $\mathrm{V}$ and PI for flow cytometric analysis. The gate setting distinguished among living (lower left), necrotic (upper left), early apoptotic (lower right) and late apoptotic (upper right) cells. (B) Quantification of the annexin $V$-positive cells as a percent of the total number of cells in the dish. Columns, mean of three experiments; bars, SE. **: $\mathrm{P}<0.01$ versus with LV-eGFP. (C) Expression of $\mathrm{p} 53$, p21Cipl/Wafl, cleaved caspase-3, 8, and 9, Bax and Bcl-2 in lentiviral vectors-transduced $\mathrm{PC} 12$ cell lines. Western blots were performed at $48 \mathrm{~h}$ post-transduction. $\beta$-actin was used as a loading control. Western blots are representative of three different experiments. (D) The relative expression levels of p53, p21, Bax and Bcl-2 in transduced PC12 cells were measure by real-time RT-PCR at $48 \mathrm{~h}$ post-transduction. All data were normalized against levels of $\beta$-actin mRNA expression within the same sample. Columns, mean from three separate experiments; bars, SE. *: $\mathrm{P}<0.05$ versus with LV-eGFP.

role of prM-Env in ZIKV induced neuronal apoptosis and further corroborates the relationship between ZIKV infection and neurological developmental disorders $[13,14,26]$.

Mechanistically, our results showed that ZIKV prM-Env, M-Env and Env overexpression elicited not only up-regulation of p53, but also changes in downstream effectors: up-regulation of p21 Cip1/Waf1, down-regulation cyclin B1 and an increase in the ratio of Bax/Bcl-2 (Figure 6). The p53 tumor suppressor is at the heart of several fundamental cellular signaling pathways. Arguably, the most important pathways for p53's tumor-suppressive role are those involved in the induction of cell cycle arrest and apoptosis [32, 33]. The cyclins are a family of proteins that control the progression of cells through the cell cycle by activating cyclin-dependent kinase (Cdk) enzymes. Cyclin B1 and Cdk1 are responsible for the transition of the cell from the $\mathrm{G} 2$ to the $M$ phase and their down-regulation will lead to G2/ M cell cycle arrest [34]. Recent evidence also suggests that p21 may participate in apoptosis in p53-dependent pathways [35]. p53-induced apoptosis results from overlapping downstream pathways that both suppress mitogenic and survival signaling and promote proapoptotic signaling. In this context, p53 can up-regulate the proapoptotic Bcl-2 family member Bax and possibly transcriptionally repress the antiapoptotic protein Bcl-2 [36]. Therefore, p53-mediated up-regulation of Bax and perhaps concomitant down-regulation of $\mathrm{Bcl}-2$ are pivotal in shifting the ratio of $\mathrm{Bax} / \mathrm{Bcl}-2$ in the cell and ultimately favoring apoptosis. 
A

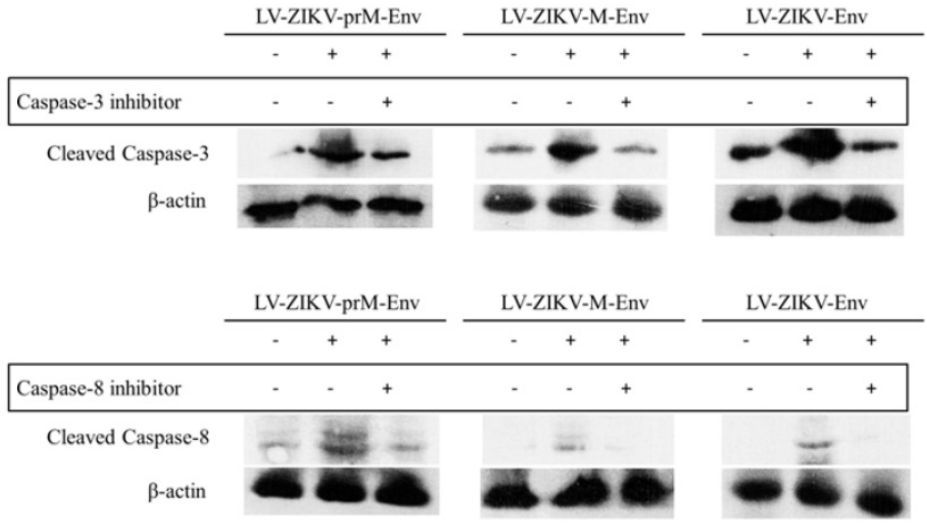

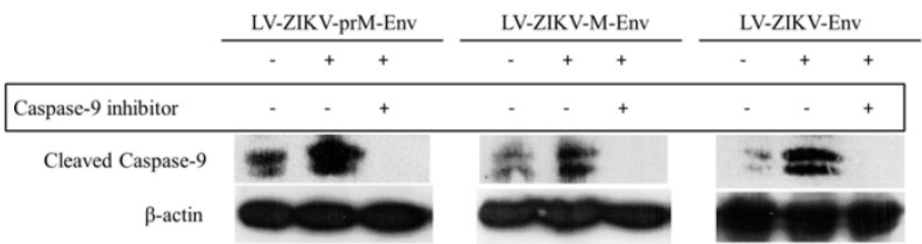

B

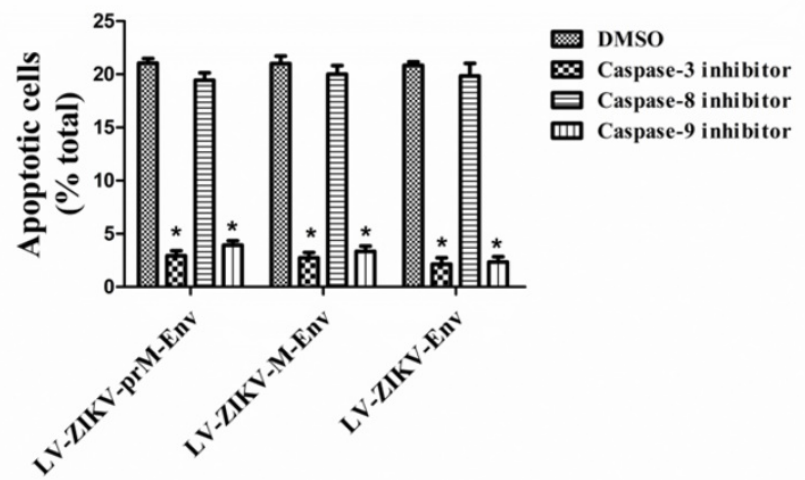

Figure 5 Effects of caspase inhibitors on ZIKV Env-mediated apoptosis in PC12 cells. (A) Cells were transduced with LV-ZIKV-prM-Env, LV-ZIKV-M-Env, LV-ZIKV-Env or LV-eGFP (100 MOI) for $6 \mathrm{~h}$ followed by treatment with either caspase-3 inhibitor Ac-DEVD-CMK $(100 \mu \mathrm{mol} / \mathrm{L})$, caspase-8 inhibitor ZIETD-FMK $(100 \mu \mathrm{mol} / \mathrm{L})$, caspase-9 inhibitor ZLEHD-FMK $(100 \mu \mathrm{mol} / \mathrm{L})$ or control solvent (DMSO) for $24 \mathrm{~h}$. Caspase-3, caspase-8 and caspase-9 were detected by western blot. (B) Treated cells were double- stained with Alexa Fluor 647 conjugated annexin $\mathrm{V}$ and PI. The annexin V-positive cells as a percent of the total number of cells were measured from flow cytometric analysis. **: $\mathrm{P}<0.01$ versus with control (DMSO-treated) cells. Data are representative of three experiments.

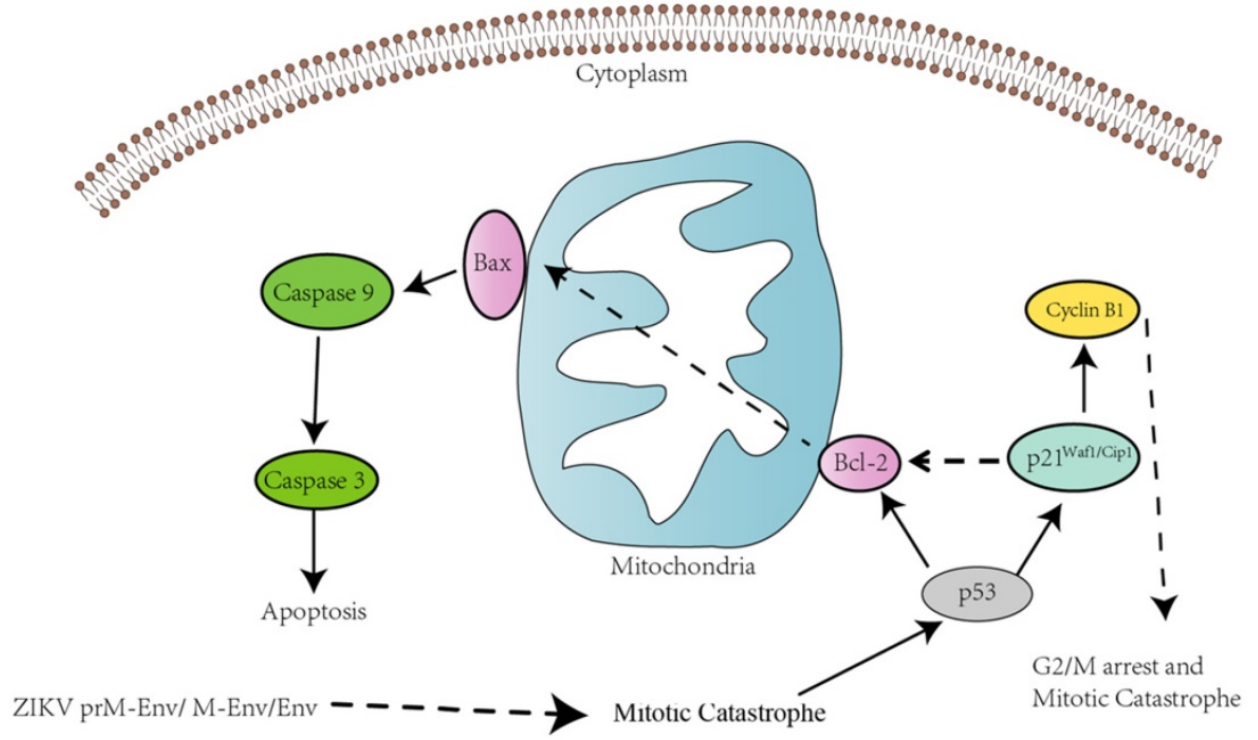

Figure 6 Schematic diagram of ZIKV Env-induced cell cycle arrest and apoptosis in PC12 cells. prM-Env, M-Env and Env induced apoptosis in lentivirus-transduced PC12 cells via an intrinsic cell death signaling pathway. P53 may involve ZIKV Env-induced G2/ M cell cycle arrest and apoptosis. 
In all, this may indicate the involvement of p53 in the G2/ M cell cycle arrest and apoptosis induced by ZIKA Env in PC12 cells. We also found that all of cleaved caspase-3, caspase-8, 9 expression levels were significantly increased in PC12 cells expressing prM-Env, M-Env or Env. However, only the inhibitors of caspase- 3 and caspase-9, not caspase-8, could block the apoptosis in these cells, which indicates that the apoptosis was induced via an intrinsic cell death signaling pathway.

\section{Acknowledgements}

We thank Dr. Junming Yue (Department of Pathology, University of Tennessee Health Science Center) for providing the vector pMD2.G and psPAX2.

\section{Author Contributions}

JL carried out lab work, analysis, and writing, QQ, XL, ZZ, AL, WH, HY, XS, XL, XY, JJ, WS, MY, YY and HY carried out lab work, and JL, HW, WW advised and contributed significantly to writing and analysis.

\section{Funding}

This study was financially supported by the National Key R\&D Projects grant 2017YFD0500600, the Industrial High-tech Fields of Science and Technology Plan Projects of Guangdong Province grant 2013B010404026, the Science and Technology Planning Project of Guangdong Province, Grant 2010A011200003, 2012B011000004, 2016A030303008, 2012B060300003, the International S\&T Cooperation Program of China grant 2011DFA33290, the Medical Scientific Research Foundation of Guangdong Province, grant A2017124, the Department of Education of Guangdong Province grant 2016KCXTD005, 2017KSYS010, the Jiangmen Introduced Innovative Scientific Research Team Program (2017).

\section{Competing Interests}

The authors have declared that no competing interest exists.

\section{References}

1. Li MI, Wong PS, Ng LC, Tan CH. Oral susceptibility of Singapore Aedes (Stegomyia) aegypti (Linnaeus) to Zika virus. PLoS neglected tropical diseases. 2012; 6: e1792.

2. Musso D, Nilles EJ, Cao-Lormeau VM. Rapid spread of emerging Zika virus in the Pacific area. Clinical microbiology and infection: the official publication of the European Society of Clinical Microbiology and Infectious Diseases. 2014; 20: O595-6.

3. Tognarelli J, Ulloa S, Villagra E, Lagos J, Aguayo C, Fasce R, et al. A report on the outbreak of Zika virus on Easter Island, South Pacific, 2014. Archives of virology. 2016; 161: 665-8.

4. Castilho A, Bohorova N, Grass J, Bohorov O, Zeitlin L, Whaley K, et al. Rapid high yield production of different glycoforms of Ebola virus monoclonal antibody. PLoS One. 2011; 6: e26040.
5. Torjesen I. Zika virus outbreaks prompt warnings to pregnant women. BMJ (Clinical research ed). 2016; 352: i500.

6. Vouga M, Musso D, Van Mieghem T, Baud D. CDC guidelines for pregnant women during the Zika virus outbreak. The Lancet. 2016; 387: 843-4.

7. Gulland A. Zika virus is a global public health emergency, declares WHO. BMJ (Clinical research ed). 2016; 352: i657.

8. Mlakar J, Korva M, Tul N, Popovic M, Poljsak-Prijatelj M, Mraz J, et al. Zika Virus Associated with Microcephaly. N Engl J Med. 2016; 374: 951-8.

9. Barreto ML, Barral-Netto M, Stabeli R, Almeida-Filho N, Vasconcelos PFC, Teixeira M, et al. Zika virus and microcephaly in Brazil: a scientific agenda. Lancet (London, England). 2016; 387: 919-21.

10. Calvet G, Aguiar RS, Melo ASO, Sampaio SA, de Filippis I, Fabri A, et al. Detection and sequencing of Zika virus from amniotic fluid of fetuses with microcephaly in Brazil: a case study. The Lancet Infectious diseases. 2016; 16: 653-60.

11. Parra B, Lizarazo J, Jimenez-Arango JA, Zea-Vera AF, Gonzalez-Manrique G, Vargas J, et al. Guillain-Barre Syndrome Associated with Zika Virus Infection in Colombia. The New England journal of medicine. 2016; 375: 1513-23.

12. Machado-Alba JE, Machado-Duque ME, Gaviria-Mendoza A, Orozco-Giraldo V. Diagnosis of neurological disorders and the Zika virus epidemic in Colombia 2014 -2016. International journal of infectious diseases: IJID: official publication of the International Society for Infectious Diseases. 2016; 51: 133-4.

13. Li C, Xu D, Ye Q, Hong S, Jiang Y, Liu X, et al. Zika Virus Disrupts Neural Progenitor Development and Leads to Microcephaly in Mice. Cell Stem Cell. 2016; 19: 120-6.

14. Souza BS, Sampaio GL, Pereira CS, Campos GS, Sardi SI, Freitas LA, et al. Zika virus infection induces mitosis abnormalities and apoptotic cell death of human neural progenitor cells. Sci Rep. 2016; 6: 39775.

15. Li H, Saucedo-Cuevas L, Shresta S, Gleeson JG. The Neurobiology of Zika Virus. Neuron. 2016; 92: 949-58.

16. Li XF, Dong $\mathrm{HL}$, Huang XY, Oiu YF, Wang HJ, Deng $\mathrm{YQ}$, et al Characterization of a 2016 Clinical Isolate of Zika Virus in Non-human Primates. EBioMedicine. 2016; 12: 170-7.

17. Zhang R, Miner JJ, Gorman MJ, Rausch K, Ramage H, White JP, et al. A CRISPR screen defines a signal peptide processing pathway required by flaviviruses. Nature. 2016; 535: 164-8.

18. Wang Y, Si LL, Guo XL, Cui GH, Fang DY, Zhou JM, et al. Substitution of the precursor peptide prevents anti-prM antibody-mediated antibody-dependent enhancement of dengue virus infection. Virus Res. 2017; 229: 57-64.

19. Sapparapu G, Fernandez E, Kose N, Bin C, Fox JM, Bombardi RG, et al. Neutralizing human antibodies prevent Zika virus replication and fetal disease in mice. Nature. 2016; 540: 443-7.

20. Li G, Poulsen M, Fenyvuesvolgyi C, Yashiroda Y, Yoshida M, Simard JM, et al. Characterization of cytopathic factors through genome-wide analysis of the Zika viral proteins in fission yeast. Proc Natl Acad Sci U S A. 2017; 114: E376-e85.

21. Liu J, Wan P, Li Q, Li X, Li A, Chen H, et al. Construction and Identification of Recombinant HEK293T Cell Lines Expressing Non-structural Protein 1 of Zika Virus. International journal of medical sciences. 2017; 14: 1072-9.

22. Zhao X, Zou Y, Gu Q, Zhao G, Gray H, Pfeffer LM, et al. Lentiviral Vector Mediated Claudin1 Silencing Inhibits Epithelial to Mesenchymal Transition in Breast Cancer Cells. Viruses. 2015; 7: 2965-79.

23. Chen X, Chen S, Pei N, Mao Y, Wang S, Yan R, et al. AAV-Mediated angiotensin 1-7 overexpression inhibits tumor growth of lung cancer in vitro and in vivo. Oncotarget. 2017; 8: 354-63.

24. Koopman G, Reutelingsperger CP, Kuijten GA, Keehnen RM, Pals ST, van Oers $\mathrm{MH}$. Annexin V for flow cytometric detection of phosphatidylserine expression on B cells undergoing apoptosis. Blood. 1994; 84: 1415-20.

25. Pinheiro TJ, Guimaraes LF, Silva MT, Soares CN. Neurological manifestations of Chikungunya and Zika infections. Arquivos de neuro-psiquiatria. 2016; 74: 937-43.

26. Wang JZ, Guo XH, Xu DG. Anatomical, animal, and cellular evidence for Zika-induced pathogenesis of fetal microcephaly. Brain \& development. 2017; 39: 294-7.

27. Fernandes NC, Nogueira JS, Ressio RA, Cirqueira CS, Kimura LM, Fernandes $\mathrm{KR}$, et al. Experimental Zika virus infection induces spinal cord injury and encephalitis in newborn Swiss mice. Experimental and toxicologic pathology: official journal of the Gesellschaft fur Toxikologische Pathologie. 2017; 69: 63-71.

28. Larocca RA, Abbink P, Peron JP, Zanotto PM, Iampietro MJ, Badamchi-Zadeh A, et al. Vaccine protection against Zika virus from Brazil. Nature. 2016; 536: 474-8.

29. Lin YC, Boone M, Meuris L, Lemmens I, Van Roy N, Soete A, et al. Genome dynamics of the human embryonic kidney 293 lineage in response to cell biology manipulations. Nature communications. 2014; 5: 4767.

30. Biederer T, Scheiffele P. Mixed-culture assays for analyzing neuronal synapse formation. Nature protocols. 2007; 2: 670-6.

31. Shaw G, Morse S, Ararat M, Graham FL. Preferential transformation of human neuronal cells by human adenoviruses and the origin of HEK 293 cells. FASEB journal: official publication of the Federation of American Societies for Experimental Biology. 2002; 16: 869-71.

32. Engeland K. Cell cycle arrest through indirect transcriptional repression by p53: I have a DREAM. Cell death and differentiation. 2017. 
33. Amulic B, Knackstedt SL, Abu Abed U, Deigendesch N, Harbort CJ, Caffrey BE, et al. Cell-Cycle Proteins Control Production of Neutrophil Extracellular Traps. Developmental cell. 2017; 43: 449-62.e5.

34. Strauss B, Harrison A, Coelho PA, Yata K, Zernicka-Goetz M, Pines J. Cyclin B1 is essential for mitosis in mouse embryos, and its nuclear export sets the time for mitosis. The Journal of cell biology. 2017.

35. Agrawal S, Agarwal ML, Chatterjee-Kishore M, Stark GR, Chisolm GM. Stat1-dependent, p53-independent expression of p21(waf1) modulates oxysterol-induced apoptosis. Molecular and cellular biology. 2002; 22: 1981-92.

36. Haldar S, Negrini M, Monne M, Sabbioni S, Croce CM. Down-regulation of bcl-2 by p53 in breast cancer cells. Cancer research. 1994; 54: 2095-7. 